\title{
Aurora A kinase inhibition enhances oncolytic herpes virotherapy through cytotoxic synergy and innate cellular immune modulation
}

\author{
Mark A. Currier ${ }^{1}$, Les Sprague ${ }^{1}$, Tilat A. Rizvi ${ }^{2}$, Brooke Nartker $^{1}$, Chun-Yu Chen ${ }^{1}$, \\ Pin-Yi Wang ${ }^{1}$, Brian J. Hutzen ${ }^{1}$, Meghan R. Franczek ${ }^{1}$, Ami V. Patel ${ }^{2}$, Katherine \\ E. Chaney, Keri A. Streby ${ }^{1,3}$, Jeffrey A. Ecsedy ${ }^{4}$, Joe Conner ${ }^{5}$, Nancy Ratner ${ }^{2}$ and \\ Timothy P. Cripe ${ }^{1,3}$ \\ ${ }^{1}$ Center for Childhood Cancer and Blood Diseases, Nationwide Children's Hospital, The Ohio State University, Columbus, \\ Ohio, USA \\ 2 Division of Experimental Hematology and Cancer Biology, Cincinnati Children's Hospital Medical Center; Cincinnati, Ohio, \\ USA \\ ${ }^{3}$ Division of Hematology/Oncology/Blood and Marrow Transplantation, Nationwide Children's Hospital, The Ohio State \\ University, Columbus, Ohio, USA \\ ${ }^{4}$ Takeda Pharmaceuticals International Co, Cambridge, MA, USA \\ ${ }^{5}$ Virttu Biologics, Ltd, Biocity, Scotland, Newhouse, United Kingdom \\ Correspondence to: Timothy P. Cripe, email: timothy.cripe@nationwidechildrens.org
}

Keywords: oHSV, Aurora A kinase, neuroblastoma, MPNST

Received: November 07, $2016 \quad$ Accepted: January 17, $2017 \quad$ Published: February 02, 2017

\section{ABSTRACT}

Malignant peripheral nerve sheath tumor (MPNST) and neuroblastoma models respond to the investigational small molecule Aurora A kinase inhibitor, alisertib. We previously reported that MPNST and neuroblastomas are also susceptible to oncolytic herpes virus (oHSV) therapy. Herein, we show that combination of alisertib and HSV1716, a virus derived from HSV-1 and attenuated by deletion of RL1, exhibits significantly increased antitumor efficacy compared to either monotherapy. Alisertib and HSV1716 reduced tumor growth and increased survival in two xenograft models of MPNST and neuroblastoma. We found the enhanced antitumor effect was due to multiple mechanisms that likely each contribute to the combination effect. First, oncolytic herpes virus increased the sensitivity of uninfected cells to alisertib cytotoxicity, a process we term virus-induced therapeutic adjuvant (VITA). Second, alisertib increased peak virus production and slowed virus clearance from tumors, both likely a consequence of it preventing virus-mediated increase of intratumoral NK cells. We also found that alisertib inhibited virus-induced accumulation of intratumoral myeloid derived suppressor cells, which normally are protumorigenic. Our data suggest that clinical trials of the combination of oHSV and alisertib are warranted in patients with neuroblastoma or MPNST.

\section{BACKGROUND}

Aurora A Kinase is a serine/threonine kinase that serves many functions in cell division, being both directly and indirectly involved with centrosome formation, spindle assembly, and cytokinesis [1-3]. Overexpression of Aurora A Kinase has been observed in multiple malignancies including bladder, breast, colon, pancreatic, malignant peripheral nerve sheath tumor (MPNST), and neuroblastoma $[1,3,4]$. Aurora A Kinase acts as an oncoprotein when transfected to target cells [5], and interestingly, Aurora A Kinase up-regulation in cancer correlates with resistance to apoptosis [6]. Due to its involvement in mitosis and association with cancer, small molecule Aurora A Kinase inhibitors, including alisertib (MLN8237), are currently under investigation as therapeutics in numerous cancer types. Multiple reports underscore the anti-tumor efficacy of alisertib in tumor models where it causes cell cycle arrest and apoptosis in cancer cells [3]. 
HSV1716 is an oncolytic herpes simplex virus (oHSV) derived from wild type HSV-1 in which the RL1 gene, encoding the protein ICP34.5 known as the "neurovirulence factor," was deleted from the viral genome [7]. In wild type HSV-1, the ICP34.5 gene product counteracts host protein kinase R (PKR) activated by the anti-viral interferon response to infection [7-9]. Without ICP34.5 expression, interferon responses in normal tissues result in PKR activation and phosphorylation of the ribosomal eIF2 $\alpha$ subunit, leading to the arrest of both protein synthesis and virus production. Many cancer types exhibit dysfunctional interferon signaling and/or activation of the MAP kinase pathway, which inhibits PKR [10], rendering cells particularly susceptible to ICP34.5-null HSV-1. Therefore, ICP34.5 deletion allows HSV1716 to selectively infect and replicate in tumor cells, while sparing healthy tissues.

Previous studies from our group and others show that a wide variety of tumor types are susceptible to infection with oHSV, including the neuralcrest-derived peripheral nerve tumors, neuroblastoma and MPNST [11-14]. Infected cells typically undergo virus-mediated cytolysis, produce immunomodulatory cytokines, and release danger signals that can lead to cell death in infected and surrounding cells [15]. While the safety, tolerability, and efficacy of HSV1716 have been demonstrated in preclinical models and several human clinical trials [1619], it is unlikely to induce cancer cures as a monotherapy. Therefore, it is of interest to utilize pharmacologic agents in combination with HSV1716 to find increasingly effective combination therapies.

Here we report that the combination of alisertib and HSV1716 is synergistic. As a mechanism for the enhanced cell killing, we found that oHSV infected cells exert a paracrine effect on uninfected cells that increases their sensitivity to alisertib. We also found that alisertib enhances virus production in tumors and slows immunemediated virus clearance by reducing innate immune cell infiltrates. Together, our data suggest that the superior outcome of HSV1716 plus alisertib in xenograft models of MPNST and neuroblastoma can be attributed to multiple diverse mechanisms.

\section{RESULTS}

\section{Alisertib and HSV1716 are synergistic in vivo}

We used models of malignant peripheral nerve sheath tumors (MPNST) and neuroblastoma because previous studies by us and others show susceptibility to alisertib and oHSV as single agents [3, 4, 11, 12, 20, 21]. Compared with vehicle controls, treatment of S462TY MPNST tumor-bearing animals with alisertib at $20 \mathrm{mg} / \mathrm{kg}$ twice daily slowed tumor growth resulting in increased overall survival (Figure 1, $p=0.0072$ ). Mice treated with HSV1716 as a single agent showed even more growth inhibition and enhanced overall survival ( $p$ $=0.0021$ ). The combination of alisertib plus HSV1716 was the most potent. We observed objective responses in all combination treated animals, including 3 partial responses (PR) and 2 complete responses (CR) and 100\% survival through day 80 (60 days after treatment ended). The effect was greater than additive as determined by the Bliss Independence Analysis of the tumor growth curves (Figure 1D). On histology, tumors showed massive necrotic areas with only small islands of viable tumor (stained with Ki67) only in the combination group (Figure 1E). All groups tolerated the dosing regimen well based on body weight change (data not shown). By using a virus inactivated by ultraviolet irradiation (UV-HSV1716), we confirmed that active virus replication is required for either single or combination efficacy (Figure 1B and 1C).

In our prior study of virus alone, some xenograft models of neuroblastoma were highly sensitive to virus, but others were less sensitive, with the most resistant being SK-N-AS [12]. Because we found SK-N-AS significantly less susceptible to virus transduction than S462TY (16\% positive versus $66 \%$ at $\mathrm{MOI}=1$ following exposure to HSV1716-GFP, respectively), we modified our treatment regimen to include more virus doses in the SK-N-AS model (Figure 2A). Interestingly, alisertib at $20 \mathrm{mg} / \mathrm{kg}$ alone was nearly as effective as alisertib plus HSV1716 in inhibiting SK-N-AS tumor growth with 7 of 8 responses (either CR, PR, or stable disease (SD)) versus 10 of 10 responses, respectively (Figure $2 \mathrm{~B}$ ). Thus in order to ascertain any combination activity, we reduced alisertib to $10 \mathrm{mg} / \mathrm{kg}$ once daily, which better mimics feasible clinical exposures, combined with repeated weekly injections of HSV1716 for a total of 3 injections. Using this regimen, either alisertib or HSV1716 alone slowed tumor growth. The combination inhibited tumor growth, an effect that lasted well beyond the end of treatment and enabled prolonged animal survival (Figure 2C and 2D). Again, the effect was greater than additive as determined by the Bliss Independence Analysis of the tumor growth curves (Figure $2 \mathrm{E}$ ). We also confirmed this reduced dose of alisertib with virus retained combination efficacy in S462TY tumors (Figure 2F and 2G).

\section{Alisertib and HSV1716 show synergistic cytotoxicity}

To study the mechanism underlying the enhanced efficacy in the alisertib plus HSV1716 cohorts, we first determined if alisertib and HSV1716 act synergistically to kill cancer cells in vitro. We exposed S462TY and SK-N-AS cells to various doses of HSV1716-GFP with or without alisertib. While each agent alone slowed cell growth, the two in combination prevented S462TY and 
SK-N-AS cell growth altogether (Figure 3A and 3B respectively). In addition, the two agents were synergistic at nearly all concentrations tested as determined by the Chou-Talalay combination index (Figure 3C and 3D). Typically, combination indices less than 0.9 indicate synergy, 0.9-1.1 indicate additivity, and greater than 1.1 indicate antagonism.

Previous reports have suggested that cells infected with HSV-1 can release byproducts that kill uninfected neighboring cells $[22,23]$. We wondered if such a paracrine signal might cause uninfected cells to be more sensitive to alisertib cytotoxicity, contributing to the synergy. To test this hypothesis, we infected cells with HSV1716-GFP and measured apoptosis (annexin-V staining) in uninfected (GFP-negative) cells. In both cell lines, virus infection caused a small increase in dead cells in uninfected cells $(<10 \%)$, consistent with previous observations of a bystander effect (Figure 4A). Furthermore, uninfected cells showed significantly more apoptosis in the cultures treated with the combination than in those treated with alisertib alone. To determine if infected cells produced a bystander effect, we also examined the effects of cultured media derived from virus-infected cells that had been filtered to eliminate residual viral particles. This conditioned media, which was supplemented $50 \%$ with normal media to ensure the presence of sufficient nutrients, was itself cytotoxic to MPNST and neuroblastoma cells and increased
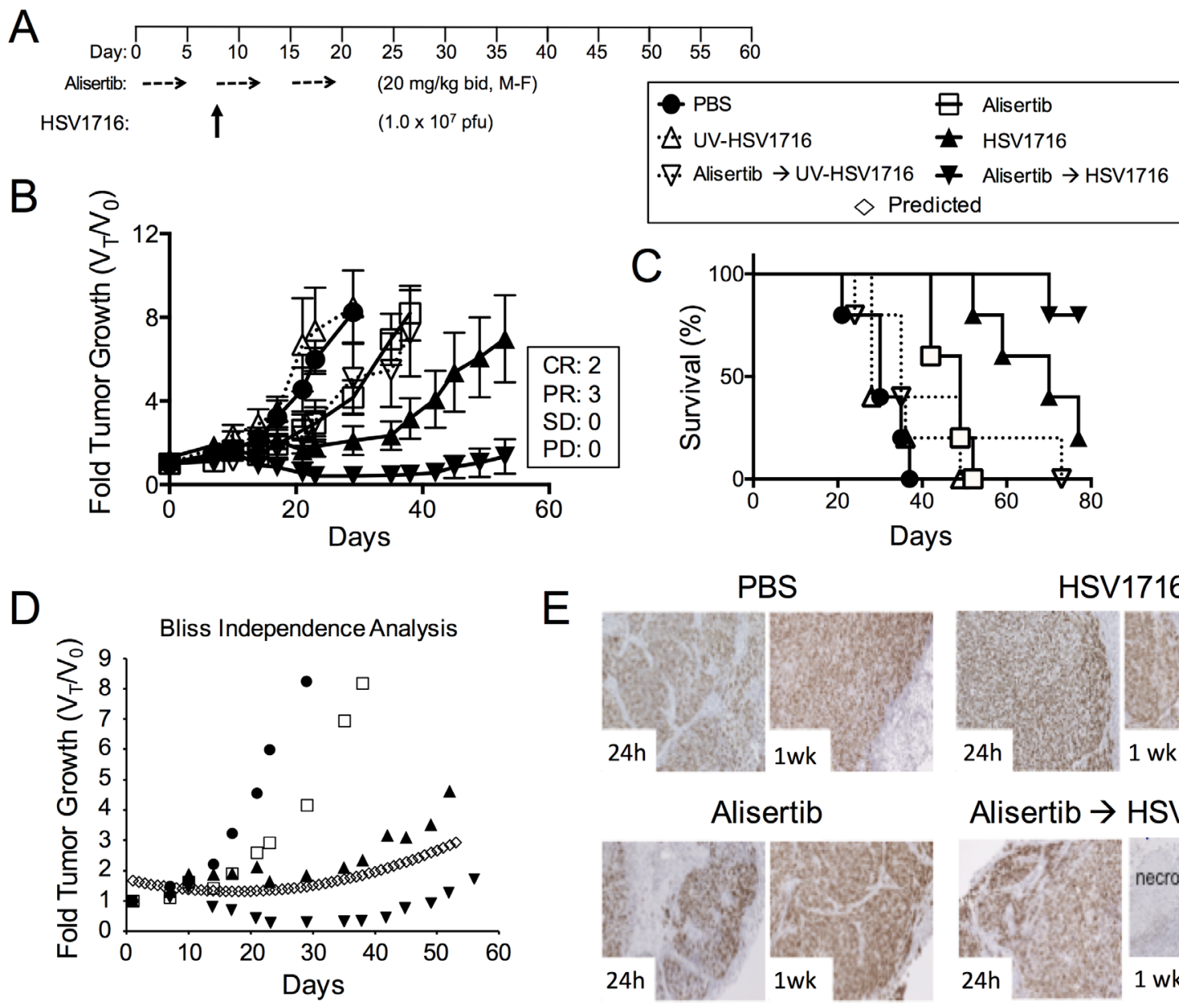

PBS

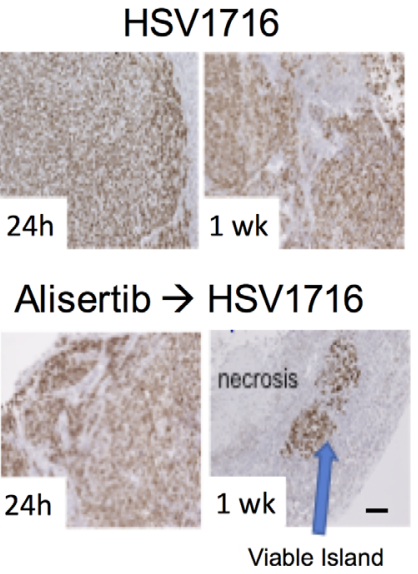

Figure 1: Alisertib and HSV1716 are synergistic in MPNST xenografts. A. Mice bearing subcutaneous S462TY xenograft tumors were given alisertib $(20 \mathrm{mg} / \mathrm{kg}$ ) or vehicle by oral gavage (twice daily 5 days per week M=Monday, F=Friday) for 19 days in addition to a single intratumoral injection of HSV1716 (1.0 x $\left.10^{7} \mathrm{pfu}\right)$, UV-HSV1716 or saline (PBS) at day $7(n=5)$. B. Tumors were measured twice per week and growth plotted as fold tumor growth. Clinical responses were categorized for each tumor in the combination group as shown complete response (CR), partial response (PR), stable disease (SD) or progressive disease (PD). C. Mice were sacrificed when the tumor volume reached $1500 \mathrm{~mm}^{3}(n=5$, $\mathrm{p}$ value for survival determined by log-rank). D. Graphical comparison of actual versus predicted (open diamonds) growth of tumors treated with combination as determined by the Bliss Independence Analysis. E. Mouse tumors were assessed by Ki67 staining at 24 hours and 1 week post infection. Areas of proliferating tumor cells were identified by Ki67 staining. Scale bar: $100 \mu \mathrm{M}$. 
alisertib cytotoxicity. We found the conditioned media from human cancer cells was less potent than that from mouse 3T6 fibroblasts (not shown), which were used for subsequent experiments (Figure 4B). The cytotoxicity due to conditioned media was due at least in part to increased apoptosis as determined by PARP cleavage, which increased compared to those observed in normal media when cells were incubated in conditioned media from virus-infected cells and exposed to different treatments (Figure 4C and 4D). These data are consistent with a paracrine effect whereby infected cells secrete signals that kill some uninfected cells and act as an adjuvant to alisertib-medited cytotoxicity. Therefore, we coined this paracrine effect virus-induced therapeutic adjuvant, or VITA.

\section{Virus pretreatment enhances synergy}

Based on this in vitro paracrine effect, we predicted that administration of virus prior to alisertib would be more effective than administration of virus given concurrently or after alisertib. Consistent with this idea, we found the tumor response to administration of alisertib and HSV1716 concurrently was slightly diminished with
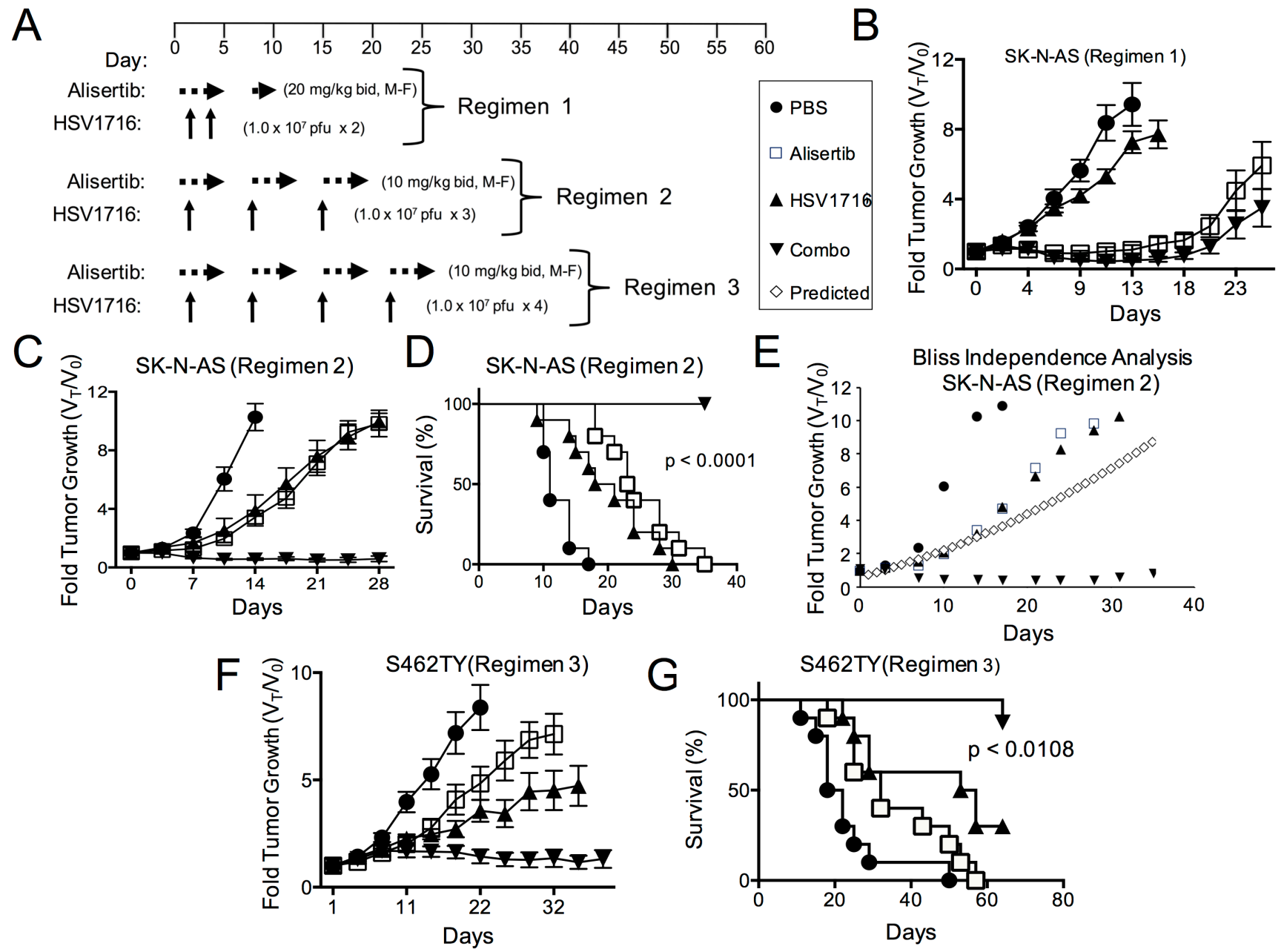

Figure 2: Alisertib and HSV1716 are synergistic in neuroblastoma xenografts. A. A schematic illustrating the multiple dosing regimens for mice bearing subcutaneous SK-N-AS or S462TY xenograft tumors. B. Mice bearing subcutaneous SK-N-AS xenograft tumors were first given alisertib $(20 \mathrm{mg} / \mathrm{kg}$ ) or vehicle by oral gavage (twice daily 5 days per week, M-F) for 9 days in addition to an intratumoral injection of HSV1716 ( 1 x $\left.10^{7} \mathrm{pfu}\right)$ or PBS on days 2 and 4. C. Mice bearing subcutaneous SK-N-AS xenograft tumors were given alisertib $(10 \mathrm{mg} / \mathrm{kg})$ or vehicle by oral gavage (twice daily 5 days per week) for 19 days in addition to intratumoral injections of HSV1716 $\left(1 \mathrm{x} 10^{7} \mathrm{pfu}\right)$ or PBS on days 5, 12 and 19. D. Survival curves of mice in panel C; mice were sacrificed when the tumor volume reached $1500 \mathrm{~mm}^{3}$ ( $n=10, p$-value for survival determined by log-rank). E. Graphical comparison of actual versus predicted growth of tumors treated with combination as determined by the Bliss Independence Analysis. F. Mice bearing subcutaneous S462TY xenograft tumors were administered alisertib $(10 \mathrm{mg} / \mathrm{kg}$ ) or vehicle by oral gavage (twice daily 5 days per week) for 26 days in addition to an intratumoral injection of HSV1716 (1.0 x 107 pfu) or PBS on days 5, 12, 19 and 26. G. Survival curves of mice in panel F; mice were sacrificed when the tumor volume reached $1500 \mathrm{~mm}^{3}$ (PBS control $(n=9)$, alisertib $(n=10)$, HSV1716 $(n=10)$ and alisertib plus HSV1716 (combo, $\left.n=8\right)$ ); p value for survival determined by log-rank) 
$1 \mathrm{CR}, 3 \mathrm{PR}$ and $1 \mathrm{SD}$ (Figure 5A) compared to the initial regimen of dosing alisertib for 5 days prior to virus, which yielded $2 \mathrm{CR}$ and $3 \mathrm{PR}$ (Figure 1B). Importantly, the mice treated with HSV1716 followed by alisertib reached CR in $80 \%$ of the mice (Figure $5 \mathrm{~B}$ ). We did not find a significant difference in overall survival between the combination cohorts (Figure 5C).

\section{Alisertib prolongs intratumoral HSV1716 viral persistence}

The mild synergy we observed in cell lines may not completely account for the large combination effect in vivo. To establish if augmented HSV1716 replication might also play a role, we measured the effect of alisertib on virus production in vitro. Alisertib caused a slight decrease of virus production over time in both tumor cell lines following virus infection (Figure 6A and 6B).
Consistent with these findings, we observed slightly decreased virus transduction in cultured cells by measuring fluorescence from GFP-expressing HSV1716 (Figure 6C and 6D). Together these results suggest that alisertib does not enhance cell autonomous virus infection kinetics.

Our findings were somewhat different using tumors grown in vivo (Figure 6E). Interestingly, the HSV1716 titer in the alisertib treated tumors peaked slightly higher at 48 hours, and the amount of recoverable infectious virus was 10 -fold above the control tumors at 96 hours. These data suggest that alisertib increases levels and persistence of HSV1716 in vivo.

\section{Alisertib prevents virus-mediated increase in intratumoral innate immune cells}

We previously observed in xenograft models of neuroblastoma that oncolytic HSV induces an intratumoral
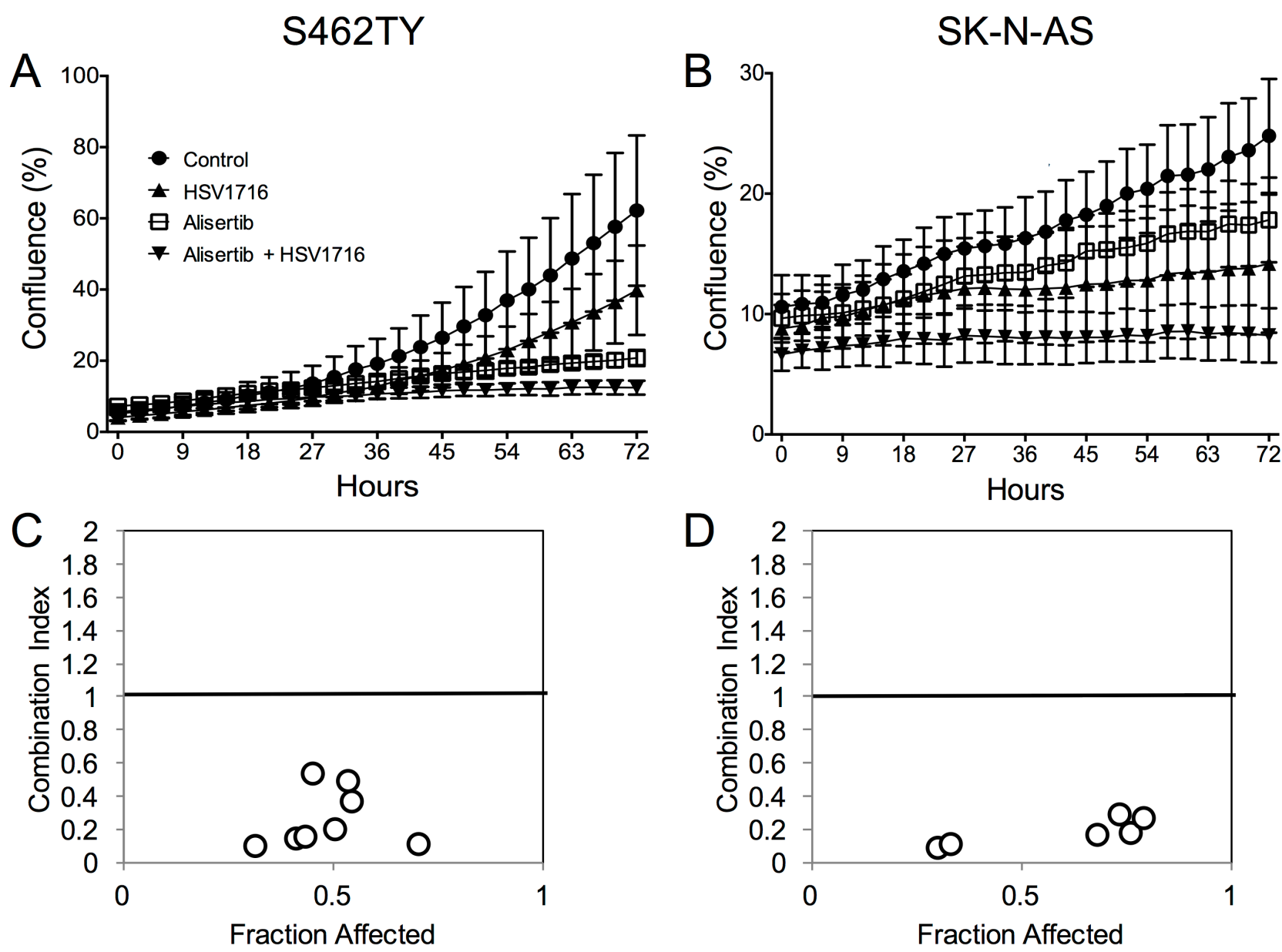

Figure 3: Alisertib and HSV1716 are synergistic in vitro. A. S462TY and B. SK-N-AS cells were treated with HSV1716-GFP $(\mathrm{MOI}=0.5)$ alone, alisertib $(50 \mathrm{nM})$ or in combination. The Incucyte ZOOM live cell imager was used to monitor cell confluence over time. C. and D. The combination index (CI) was determined by titrating HSV1716-GFP (MOI = 0, 0.01, 0.1, 1, 10) with alisertib $(0,15,31$, $62,125,250,500,1000 \mathrm{nM}$ ) and synergy was calculated using the Chou-Talalay method of synergy analysis with the Compusyn Software and then $\mathrm{CI}$ value plotted versus the fraction affected. For the in vitro assays, all data points represent mean $\pm \mathrm{SD},(n=4$ measurements per well). All the experiments were repeated 3 times. Representative data are shown. 
infiltration of innate inflammatory cells (unpublished). Because alisertib increased HSV1716 kinetics in vivo but not in vitro, we postulated that alisertib might alter the tumor immune microenvironment. In S462TY tumors we found that virus alone increased intratumoral Ly $6 \mathrm{c}^{\mathrm{hi}}$ monocytic myeloid derived suppressor cells (mMDSC), natural killer $(\mathrm{NK})$ cells, and Ly6ghi tumor associated neutrophils (gMDSC) (Figure 7). Interestingly, alisertib prevented the increase in mMDSCs and NK cells without affecting virus-induced changes in neutrophils or macrophages. Fewer NK cells following virus infection as a result of alisertib treatment may explain the higher and more persistent virus titers we found in tumors.

\section{DISCUSSION}

Alisertib is a viable clinical therapeutic shown to be safe in single agent and combination studies. Additionally, alisertib is an effective clinical therapeutic shown to induce stable disease and partial responses in phase I and
II studies [24, 25]. Alisertib is of particular interest for neuroblastoma due to preclinical efficacy in xenograft models [21] and the discovery that Aurora A Kinase stabilizes the oncogene MYCN [26, 27]. Phase I studies in children showed that alisertib is safe both as a single agent and in combination with chemotherapy. Some patients in these early phase trials experienced stable disease and partial responses across several different tumor types [28, 29]. As a single agent, alisertib also produced prolonged stable disease in some patients with MPNST [30].

Talimogene Laherparepvec (T-VEC) is a modified herpes virus that recently was given FDA approval for melanoma by intralesional injection based on favorable phase III clinical trial data [31]. HSV1716, like T-VEC, is attenuated by deletion of the ICP34.5 genes, and shows widespread cytolytic activity against a variety of cancer types, including neuroblastoma [12]. Other herpes virus mutants have also been shown to have activity in preclinical models of neuroblastoma [11, 32] and MPNST [13, 14, 33-35]. HSV1716 demonstrated safety in several clinical trials after delivery by intracranial and
A

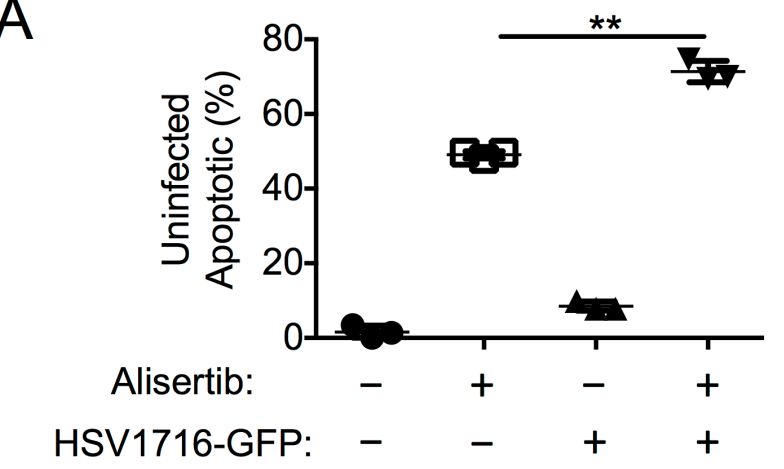

C

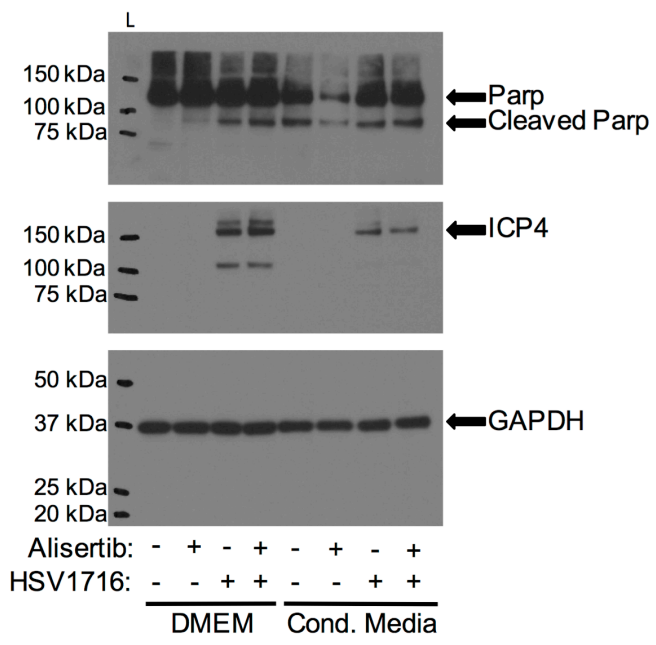

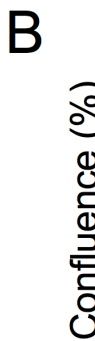

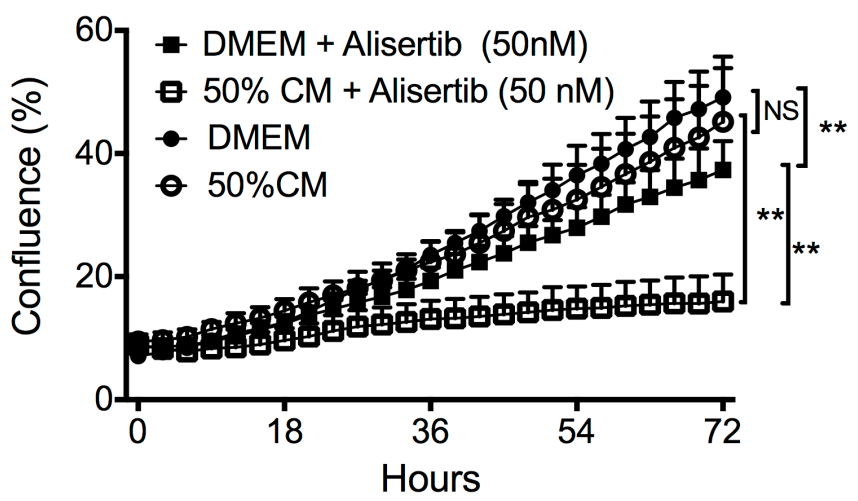

D

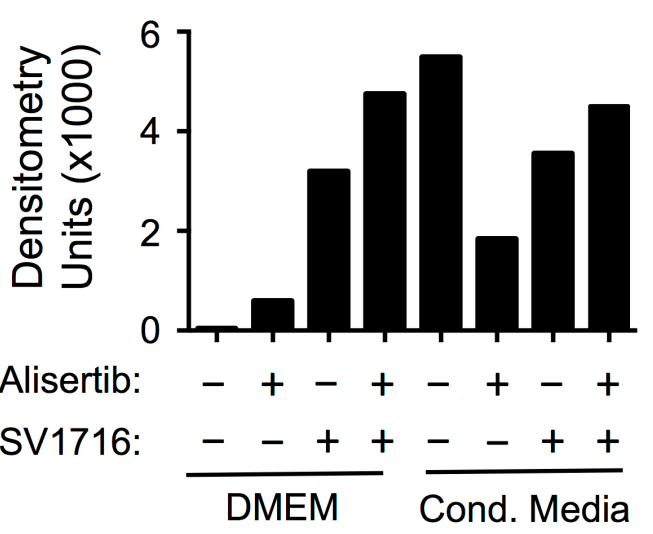

Figure 4: Virus-Induced Therapeutic Adjuvant (VITA) sensitizes target cells to alisertib therapy. A. Apoptosis (Annexin $\mathrm{V}$ positive by flow cytometry) in uninfected S462TY cells identified by GFP negativity in cultures exposed to HSV1716-GFP and/or alisertib. B. Effect of 50\% conditioned media (CM) from HSV-infected cells on S462TY sensitivity to alisertib. C. Western blots of cleaved PARP and virus ICP4 expression in S462TY cell lysates exposed to alisertib and/or HSV1716 when grown in DMEM or Conditioned Media. D. Densitometry of cleaved PARP for each sample in the blot shown in (C). ${ }^{* *} P<0.01$ as determined by 2 -way ANOVA. 
intralesional injections [16-19]. A phase I safety trial in children and young adults is also currently underway (www.clinicaltrials.gov NCT00931931). As with most cancer therapies, however, combination therapies may be required to fully leverage the anti-tumoral efficacy of oncolytic herpes simplex viruses.

We found that inhibition of Aurora A Kinase activity with alisertib used in combination with oncolytic herpes virus improves antitumor efficacy in neurogenic tumor models. The combination effect was due in part to synergistic cytotoxicity. Synergy appears to result from a bystander effect that renders uninfected cells more susceptible to alisertib. In vivo, alisertib slows virus clearance from tumors, likely due to mitigation of virusinduced NK cells that would normally be expected to kill virus-infected cells, prolonging oncolysis and contributing to antitumor efficacy. Indeed, the NK cell response was shown to diminish virolytic effects in preclinical models of brain tumors [36]. In addition, alisertib had other effects on the tumor immune microenvironment, including the prevention of virus-induced mMDSC accumulation. The effect is likely due to a systemic inhibition of proliferation of these cells because we also observed inhibition of virus-induced mMDSC and NK cells in spleens. The prevention by alisertib of an increase in mMDSC that normally accompanies virus infection may also improve
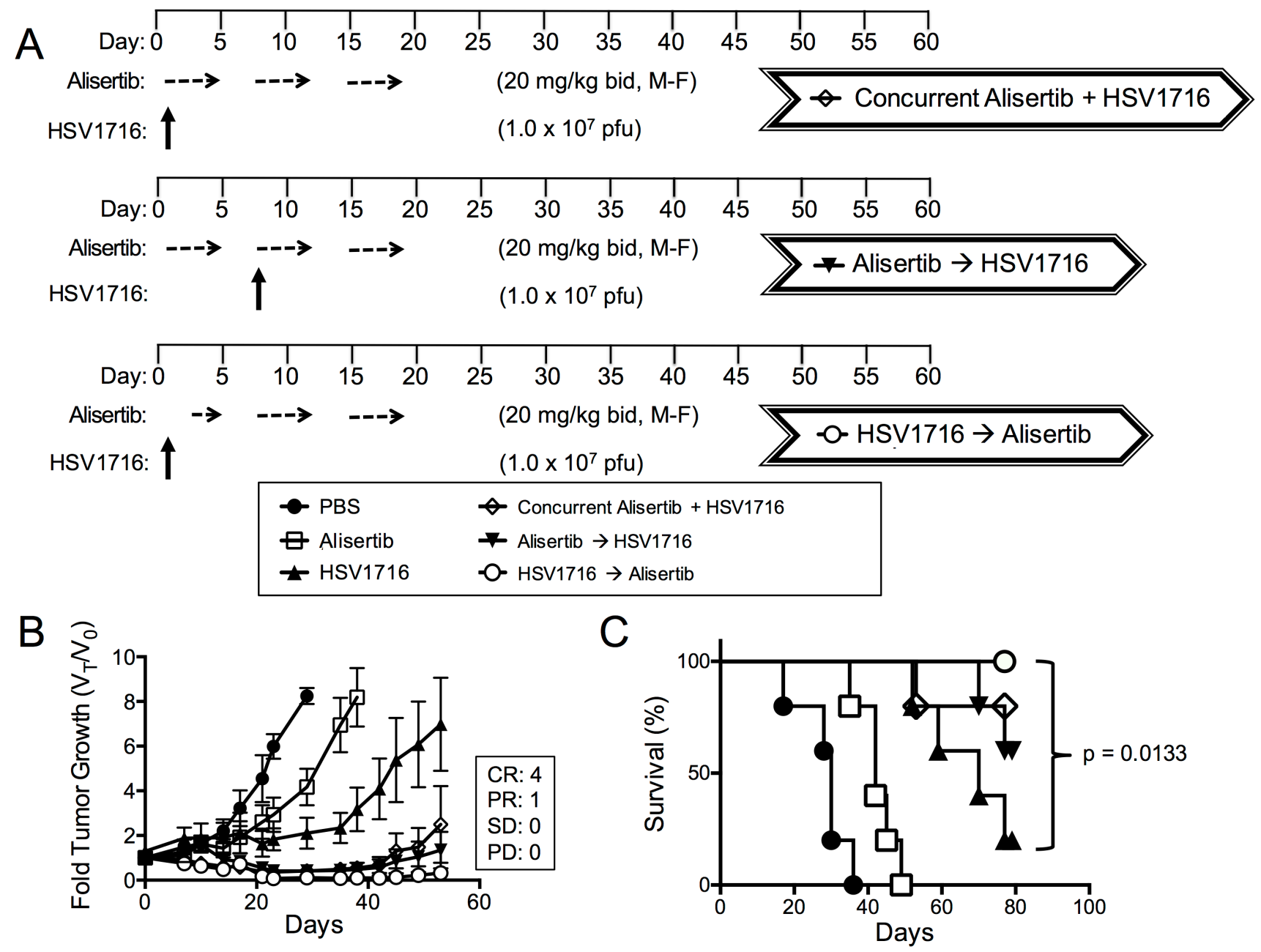

40
$M-F)$
Alisertib

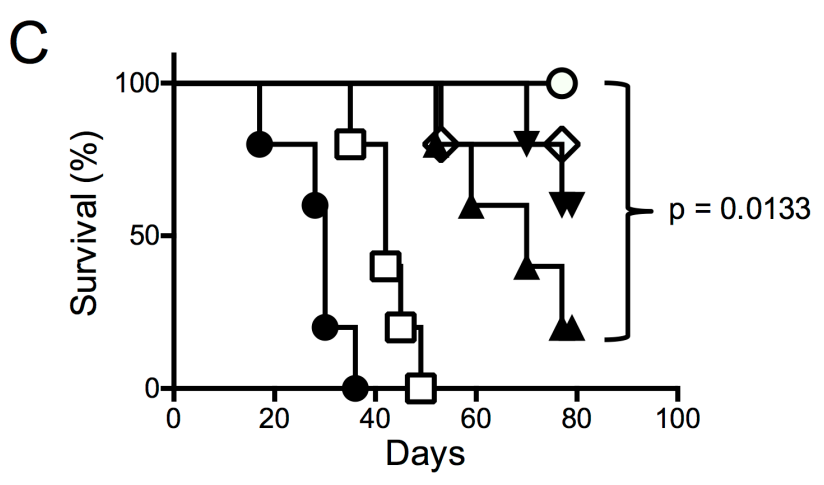

Figure 5: Virus pretreatment enhances synergy. A. Dosing schema for mice bearing subcutaneous S462TY xenograft tumors. Mice were first given alisertib $(20 \mathrm{mg} / \mathrm{kg}$ ) or vehicle by oral gavage (twice daily 5 days per week, M-F) for 19 days in addition to an intratumoral injection of HSV1716 $\left(1 \times 10^{7} \mathrm{pfu}\right)$ or PBS on day 1 . Another cohort of mice bearing subcutaneous xenograft tumors received alisertib $(20$ $\mathrm{mg} / \mathrm{kg}$ ) or vehicle by oral gavage (twice daily 5 days per week) for 19 days in addition to an intratumoral injection of HSV1716 (1 x $10^{7}$ pfu) on day 5. A final cohort of mice bearing subcutaneous S462TY xenograft tumors were administered a single injection of HSV1716 (1 x $\left.10^{7} \mathrm{pfu}\right)$ on day 1 followed by alisertib $(20 \mathrm{mg} / \mathrm{kg})$ initiating on day 3 by oral gavage (twice daily for, 5 days per week) for a period 16 days. B. S462TY tumors were measured twice per week and growth plotted as fold tumor growth. Clinical responses were categorized for each tumor in the combination group as shown complete response (CR), partial response (PR), stable disease (SD) or progressive disease (PD). C. Survival curves of mice in panel B; mice were sacrificed when the tumor volume reached $1500 \mathrm{~mm}^{3}(n=5$, $\mathrm{p}$ value for survival determined by log-rank). 

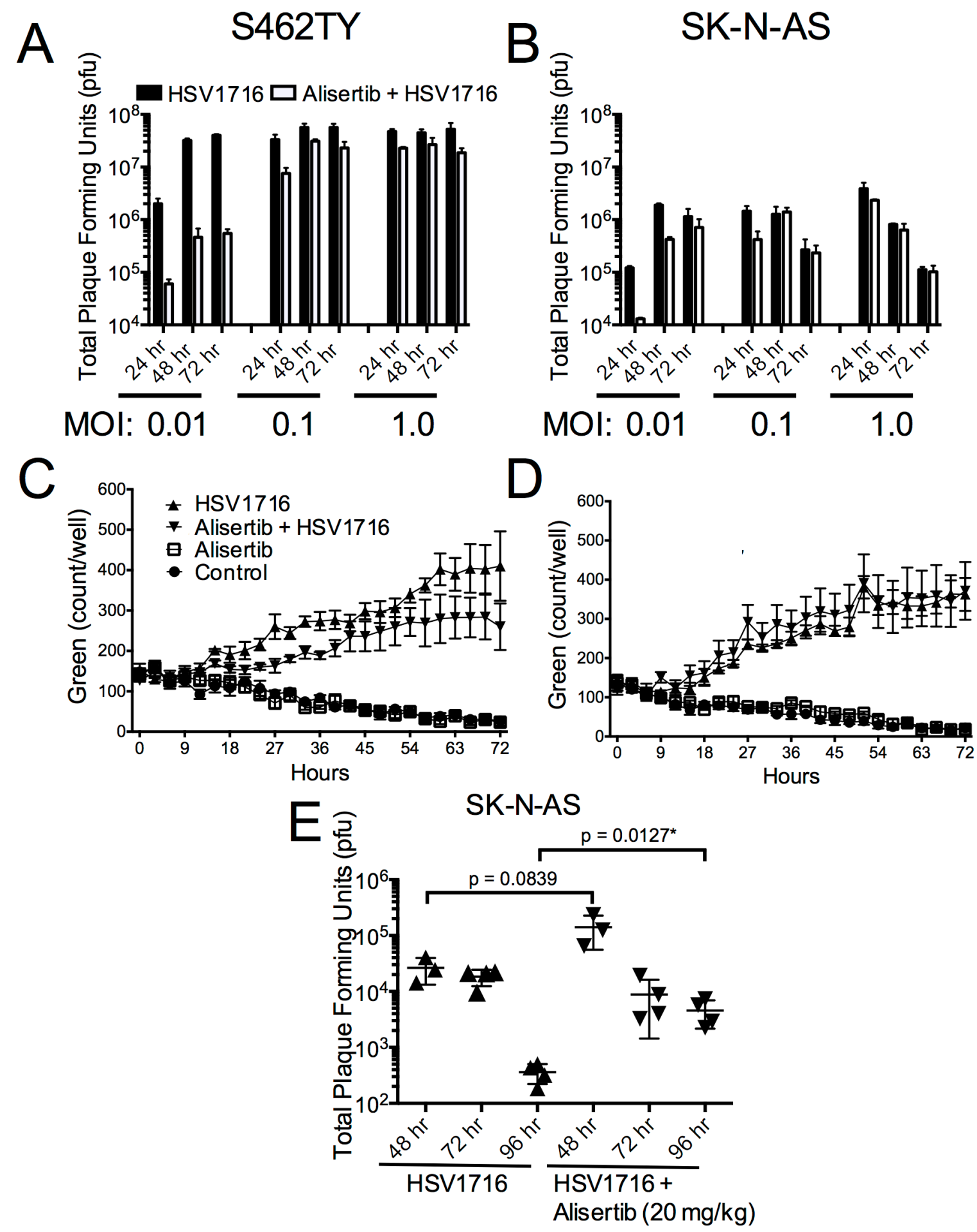

Figure 6: Alisertib decreases virus replication in vitro but enhances HSV1716 replication and persistence in vivo. A. S462TY tumor cells were treated with alisertib $(80 \mathrm{nM})$ or vehicle for 48 hours and then infected with HSV1716 at the various MOI indicated. Cells were harvested at 24, 48 and 72 hours post virus injection and analyzed for virus replication by plaque assay. For the in vitro replication assays, all data points represent mean $+\mathrm{SD},(n=4$ replicates per MOI at each time point). C. and D. S462TY or SK-N-AS tumor cells were treated with alisertib $(80 \mathrm{nM})$ or vehicle for 48 hours and then infected with HSV1716-GFP (MOI 0.1). Green fluorescence was monitored over time using the Incucyte. For the in vitro cytotoxicity assays, all data points represent mean $\pm \mathrm{SD},(n=4$ measurements per well). All the experiments were repeated 3 times. Representative data are shown. E. Mice bearing SK-N-AS xenograft tumors were treated with alisertib (20 mg/kg twice daily) or vehicle and given a single intratumoral injection of $1 \times 10^{7}$ pfu HSV1716, and the amount of infectious virus recovered at various times determined by plaque assay. For the in vivo replication studies, all data points represent mean $\pm \mathrm{SD},(n=3-4$ replicates per time point). 
cancer virotherapy, as mMDSC are known to convert to pro-tumorigenic M2-like macrophages [37]. Our multimechanistic model for the effect of combination alisertib with herpes virotherapy is illustrated in Figure 8.
The mechanisms by which oncolytic viruses induce tumor shrinkage extend beyond lytic infection to include an adaptive immunotherapeutic component [15]. Virus infection can increase recruitment and activation of

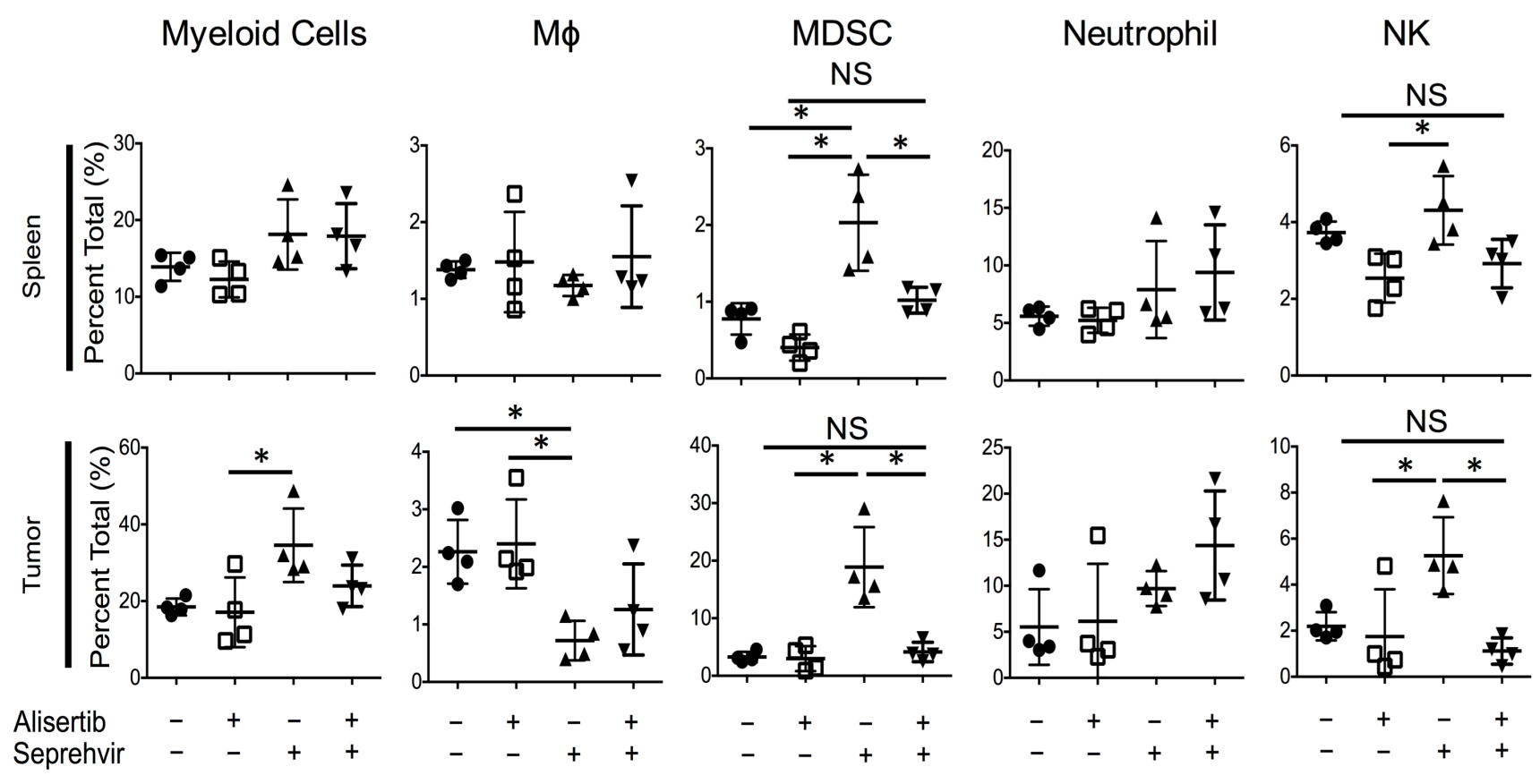

Figure 7: Alisertib changes virus-induced immune cellular composition. Mice bearing S462TY xenograft tumors were treated with alisertib $\left(20 \mathrm{mg} / \mathrm{kg}\right.$ twice daily) or vehicle and given a single intratumoral injection of 1 x $10^{7}$ pfu HSV1716. Tumors and spleen were harvested 3 days later and immune cell compositions were quantified by single cell flow cytometry. Myeloid Cells: CD11 ${ }^{+}$cells in live

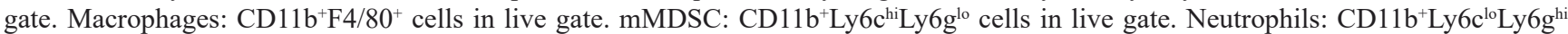
cells in live gate. NK cells: F4/80-CD49B ${ }^{+}$cells in live gate. For the in vivo cellular infiltrate studies, all data points represent mean $\pm \mathrm{SD}(n$ $=4$ replicates). Experiments were repeated 2 times.

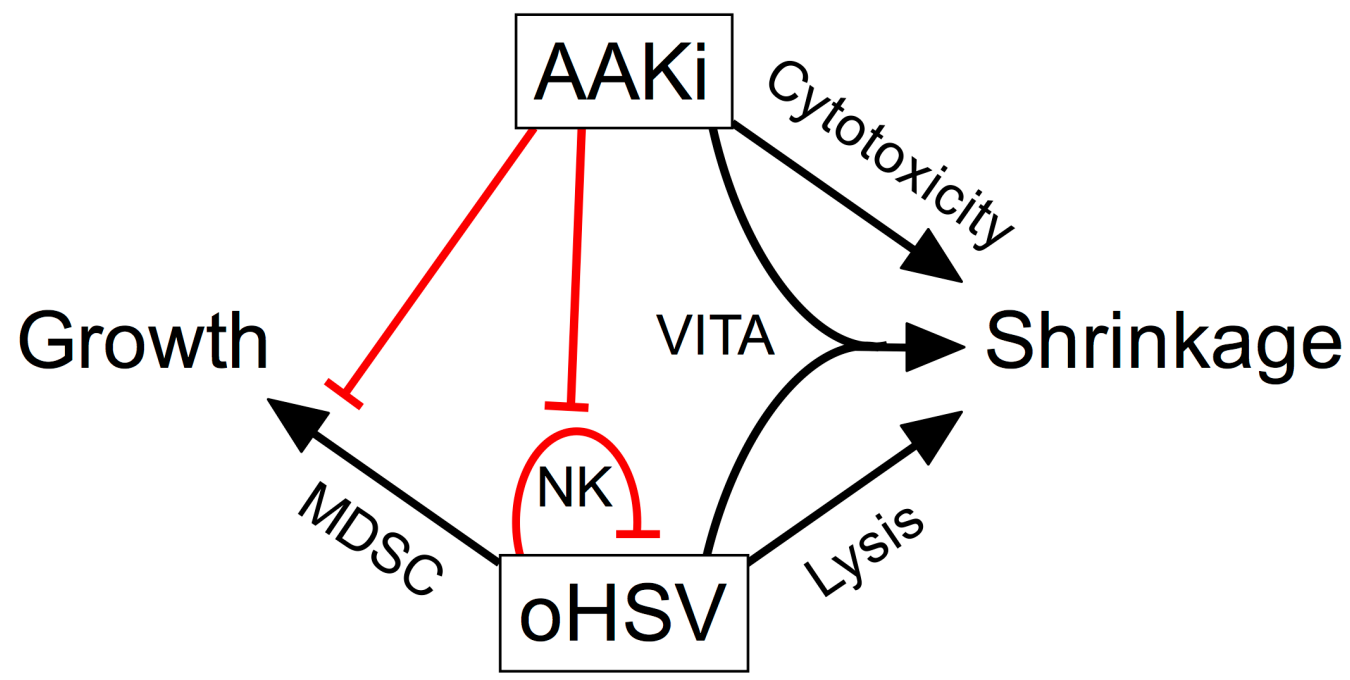

Figure 8: Model of pleotropic effects of alisertib on oncolytic herpes virotherapy. Our data suggest a model whereby inhibition of aurora A kinase combines with virotherapy in multiple ways to elicit tumor shrinkage. Alisertib causes direct cytotoxicity and virus induces direct cell lysis. In addition, virus infection sensitizes surrounding cells to alisertib, an effect termed virus-induced therapeutic adjuvant (VITA). Virus infection also induces NK and mMDSC infiltration and/or proliferation, which is prevented by alisertib. The loss of NK cells slows virus clearance, enabling more prolonged oncolysis. The prevention of mMDSCs may also play a role as these cells can differentiate into M2-like macrophages and promote tumor growth. 
antitumor T cells, broaden the tumor antigens targeted by $\mathrm{T}$ cells [38], and synergize with $\mathrm{T}$ cell checkpoint inhibitors [39]. Because Aurora A Kinase is important in T cell proliferation and its inhibition impairs $\mathrm{T}$ cell expansion [40], one potential downside may be that alisertib could impair the immunotherapeutic effects of HSV1716, which we would not have detected in our studies using immunodeficient mice. In that regard, however, it is interesting to note that the secretion of CCL5 induced by alisertib (so-called senescence-associated secretory phenotype) in other cancer models actually increased rather than decreased tumor infiltrating lymphocytes [41]. Because most animal cells are not highly permissive to human herpes simplex virus infection, the full impact of alisertib on the integrated effects of both lytic and immunotherapeutic phases will only truly be testable through human trials.

The effect of alisertib on virotherapy may not be limited to herpes virus, as the combination of alisertib also improves the antitumor efficacy of oncolytic measles virus in several cancer models [42]. In those studies, increased virus gene expression was suggested to be a possible mechanism of interaction. Alisertib also induced expression of cellular genes, such as IL-24, which they postulated might increase antitumor effects.

Two reports using HSV-1 derivatives are consistent with our observation of a bystander effect. Stanziale and colleagues noted that virus infection increased apoptosis in uninfected cells and limited virus spread, which was reversed with an inhibitor of apoptosis [22]. Takasu et al. noted oHSV infection induced a so-called immunogenic cell death with secretion of damage associated molecular patterns ATP and HMGB1, and that intratumoral injection of supernatants from infected cells slowed tumor growth [23]. Although the exact nature of molecules that mediate the bystander effect are not known, it is possible this phenomenon will sensitize cells not only to alisertib but also to other targeted or cytotoxic drugs. Whether the bystander effect occurs with other viruses is unknown, but there are reports of other viruses synergizing with chemotherapy or targeted small molecules [43, 44].

Alisertib did not increase oHSV permissivity in vitro. In fact, under most conditions the combination of alisertib with oHSV reduced virus production. We attribute this finding to drug-induced senescence and cell death resulting in fewer cells available for infection. It is also possible that alisertib actually impairs virus production at the cellular level. In this regard, it is interesting to note that aurora A kinase regulates NuMA phosphorylation [45]. NuMA is a nuclear matrix protein whose cellular localization is determined by phosphorylation status and is essential for HSV assembly and egress [46]. In contrast, several other small molecules including the proteasome inhibitor bortezomib [47], doxorubicin [48], a copper chelator [49], and HDAC inhibitors [50, 51] synergize with oHSV by increasing herpes virus production. Thus multiple mechanisms exist by which co-administered drugs may enhance oncolytic herpes virotherapy.

In summary, we found that aurora A kinase inhibition by alisertib synergizes with oncolytic herpes virotherapy, likely through multiple mechanisms. Our results support testing of this combination of agents, particularly in children and young adults with neuroblastoma and MPNST.

\section{MATERIALS AND METHODS}

\section{Compounds and reagents}

Alisertib (a.k.a. MLN8237) was generously provided by Millennium Pharmaceuticals, a wholly owned subsidiary of Takeda Pharmaceutical Company Limited (Boston, MA). For in vitro studies, alisertib was dissolved in dimethyl sulfoxide (DMSO, Sigma Aldrich, St. Louis, MO) at $50 \mathrm{mM}$ and then diluted to the appropriate concentrations in complete cell culture media. DMSO alone was diluted in complete cell culture media as a control for the in vitro assays. For in vivo studies, alisertib was formulated daily at 2.5 or $5.0 \mathrm{mg} /$ $\mathrm{mL}$ in 3\% ethanol / 9.7\% 2-hydroxypopyl-b-cyclodextrin / 0.97\% sodium bicarbonate. The monoclonal antibodies anti-CD49b-PE (Clone DX5), anti-F4/80-PECy7 (Clone BM8), anti-Ly6G-APC-Cy7 (Clone 1A8) and anti-CD11bViolet 421 (Clone M1/70) were purchased from Biolegend (San Diego, CA). Monoclonal antibody anti-Ly6C-APC (AL-21) was procured from BD Biosciences (San Jose, CA). Anti- poly-ADP-ribose polymerase (11835238001) antibody was purchased from Roche Indianapolis, IN). Anti-ICP4 (Clone 10F1) antibody was obtained from Virusys Corporation (Taneytown, MD), and anti-GAPDH (Clone 14C10) antibody was obtained from Cell Signaling Technology (Danvers, MA).

\section{Viruses}

HSV1716 (HSV1716) and HSV1716-GFP, an engineered mutant that expresses green fluorescent protein under the control of the CMV promoter, were generously provided byVirttu Biologics (Glasgow, U.K.). HSV1716 is derived from the HSV-1 strain $17+$, but has been engineered with a deletion of the gene encoding ICP34.5, reducing its virulence and causing it to be permissive for replication selectively in rapidly dividing cells $[52,53]$. The non-replicating virus control, UV-HSV1716, was prepared by exposing a vial of HSV1716 to a germicidal ultraviolet light. Briefly, $1.5 \mathrm{~mL}$ of HSV1716 $\left(5 \times 10^{8} \mathrm{pfu} /\right.$ $\mathrm{mL}$ ) was placed in an open petri dish on ice in a Nuaire Labgard Class II, Type A2 Laminar Flow Biological Safety Cabinet and directly exposed to the germicidal ultraviolet light at 253.7 manometers with an average 
intensity of 100 microwatts per centimeter for 45 minutes. The UV-inactivated HSV1716 sample was then confirmed by standard viral plaque assay to be negative for plaque forming units ( $\mathrm{pfu}$ ) prior to experimental use.

\section{Cell lines and cultures}

The history and cell culture maintenance of tumor cell lines S462TY, a human malignant peripheral nerve sheath tumor, and SK-N-AS, a human neuroblastoma, were previously reported $[12,33]$. Briefly, S462TY and SK-N-AS cells were grown in Dulbecco's modified essential media (DMEM) supplemented with $10 \%$ fetal bovine serum (FBS) and 1\% penicillin/streptomycin. In addition, all cells were cultured at $37^{\circ} \mathrm{C}$ in a humidified incubator at $5 \% \mathrm{CO}_{2}$. Both cell lines were subjected to routine short tandem repeat genotyping analysis (Molecular Genetics Core, Nationwide Children's Hospital, Columbus, $\mathrm{OH}$ ) and mycoplasma testing using the MycoAlert ${ }^{\mathrm{TM}}$ Mycoplasma Detection Kit (LT07-318, Lonza Inc. Allendale, NJ).

\section{Preparation of conditioned media}

3 T6 mouse fibroblasts were used to produce HSV1716-conditioned media. Briefly, 3T6 cells were allowed to reach $50 \%$ confluence, washed one time with phosphate buffered saline (PBS), and fresh DMEM (10\% FBS, 1\% penicillin/streptomycin) was added. 3 T6 cells were then infected by a MOI of three with HSV1716, UV-inactivated HSV1716, or PBS and incubated for $24 \mathrm{~h}$ at $37^{\circ} \mathrm{C} 5 \% \mathrm{CO} 2$ under sterile conditions. Supernatants were collected and centrifuged at 4,000 g for 10 minutes. Supernatants were then filtered using the SteriFlip vacuum-driven filtration system $(0.22 \mu \mathrm{m}$, EMD Millipore, Billerica, MA). Finally, supernatants were then UV-irradiated for 45 minutes on ice to inactivate any remaining competent virus. All supernatants were confirmed to be virus-free by standard plaque forming assay.

\section{Immunohistochemistry}

Formalin fixed tissues were paraffin embedded, sectioned at $5 \mu \mathrm{M}$ thickness, baked at $60^{\circ} \mathrm{C}$ for 1 hour and air dried. We deparaffinized, hydrated, transferred sections to $0.1 \mathrm{M}$ citrate buffer $(\mathrm{pH}$ 6.0) for antigen retrieval, boiled for 10 minutes, then cooled at room temperature for 30 minutes. Sections were quenched with $3 \%$ hydrogen peroxide for 10 minutes, rinsed in PBS, and blocked in normal goat serum (10\% serum with $0.3 \%$ Triton-X-100 in PBS) for 1 hour. Then, sections were incubated overnight in primary antibody diluted in blocking solution. We used rabbit anti-Ki67 (Novocastra; 1:5,000). After PBS rinses, sections were incubated in biotinylated goat anti-rabbit secondary antibodies (Abcam, Cambridge, MA) for 1 hour at room temperature, rinsed in $\mathrm{PBS}$, incubated in $\mathrm{ABC}$ reagent (Vector Laboratories, Burlingame, $\mathrm{CA}$ ) for one hour, rinsed in PBS and incubated in DAB (Vector Laboratories) for 5 minutes. After DAB, sections were rinsed, dehydrated in series of graded alcohols $(70 \%$, $95 \%$, and $100 \%$ ), cleared in Xylenes and finally cover glassed in Histomount (Life Technolgies, Carlsbad, CA). We acquired microscopic images with Openlab software suites on a Zeiss Axiovert 200.

\section{In vitro synergy assays}

To determine two-drug synergy in vitro, cells were seeded at 2,000 cells per well in a 96 flat bottom well plate and allowed to attach overnight. Cells were then washed with sterile PBS. Fresh media was added containing HSV1716-GFP (MOI = 0, 0.01, 0.1, 1, 10) alone, alisertib $(0,15,31,62,125,250,500,1000 \mathrm{nM})$ alone or the combination of the HSV176-GFP and alisertib at the various MOI and concentrations, respectively. Cell proliferation was monitored by phase contrast imaging every 3 hours using the IncuCyte Zoom time lapse imaging system (Essen Biosciences, Ann Arbor, MI, USA). Synergy was determined using the Chou-Talalay method of synergy analysis [54] using CompuSyn for Drug Combinations Analysis Software (ComboSyn, Inc., Paramus, NJ, USA).

\section{In vitro virus replication}

Tumor cells were seeded in 12-well tissue culture dishes at a concentration of $1.75 \times 10^{5}$ cells per well and incubated overnight at $37^{\circ} \mathrm{C}$ in a humidified incubator at $5 \% \mathrm{CO}_{2}$. After replacing the culture media with fresh media containing alisertib at 0 or $80 \mathrm{nM}$, the cells were then incubated for an additional 48 hours. Prior to infecting the cells with virus, representative control and alisertib treated cells were counted by trypan blue exclusion to determine the appropriate virus infection concentration. After washing cells and adding fresh media containing alisertib at 0 or $80 \mathrm{nM}$, cells were infected with HSV1716 at a multiplicity of infection $(\mathrm{MOI})=0.01,0.1$ and 1.0 . Infected cells were harvested at 24, 48 and 72 hours post virus infection by cell scraping. Harvested cells and media were freeze-thawed three times and then assayed for total plaque forming units via standard plaque assay.

\section{Cell permissivity assay}

To monitor virus infection and spread, S462TY or SK-N-AS cells were seeded at 2,000 cells per well in a 96 flat bottom well plate and allowed to attach overnight. 
Cells were washed with sterile PBS, then either fresh media or conditioned media (prepared from virus-infected cells, see above) was then added to each well and treated with or without HSV1716-GFP (MOI $=0.5)$ in the presence or absence of alisertib (50 nM). Virus infection was monitored by phase contrast and GFP-imaging every three hours using the IncuCyte Zoom time lapse imaging system (Essen Biosciences, Ann Arbor, MI, USA).

\section{Animal studies}

All animal studies were approved by the Institutional Animal Care and Use Committee (IACUC) for the Research Institute at Nationwide Children's Hospital. Female athymic nude mice, age 4-6 weeks and weighing 15-25 g were purchased from Envigo (Indianapolis, IN). These mice were subcutaneously injected with $5.0 \times 10^{6}$ S462TY or $1.0 \times 10^{7} \mathrm{SK}-\mathrm{N}-\mathrm{AS}$ cells in $150 \mu \mathrm{L}$ mix of phosphate-buffered saline (PBS) and matrigel (2:1). Alisertib was formulated at 2.5 or $5 \mathrm{mg} / \mathrm{mL}$ in the vehicle described above. Alisertib or vehicle was administered by oral gavage in $100 \mu \mathrm{L}$. HSV1716 was diluted in PBS to $1.0 \times 10^{8} \mathrm{pfu} / \mathrm{mL}$ and administered intratumorally in a final volume of $100 \mu \mathrm{L}$. PBS was administered intratumorally as the control for HSV1716 in a final volume of $100 \mu \mathrm{L}$.

\section{Efficacy studies}

When the tumors reached a mean volume between $175-250 \mathrm{~mm}^{3}$, the mice were randomized into four study groups: vehicle + PBS, alisertib + PBS, vehicle + HSV1716 and alisertib + HSV1716. The alisertib dosing schema and dose was varied to determine the most efficacious regimen and if a clinically relevant dose could be achieved. UV- HSV1716 was used in a control to determine if virus replication was essential. The specific dosing schema is described for each experiment in the figure legends. Animals were followed until the animal reached endpoint criteria, including tumor volume > $1500 \mathrm{~mm}^{3}$, body weight loss $>20 \%$, unusual mouse behavior, lack of movement or poor posture. Tumor size was measured using calipers twice per week and tumor volume was calculated using the following formula: $\mathrm{a} \times \mathrm{b} 2(\pi / 6)$ where $\mathrm{a}$ is the longest diameter and $\mathrm{b}$ is the shortest diameter. Mice were also weighed and observed 2 times per week for signs of endpoint condition. Mice that demonstrated signs of toxicity or reached endpoint criteria were humanely euthanized by $\mathrm{CO}_{2}$ asphyxiation and subjected to cervical dislocation as the secondary method of euthanasia.

\section{Virus replication studies}

When the tumors reached a mean volume $>400$ $\mathrm{mm}^{3}$, the mice were randomized into four study groups:
PBS + vehicle, PBS + alisertib, HSV1716 + vehicle and HSV1716 + alisertib. A single dose of HSV1716 was injected intratumorally on day 0 followed by oral gavage administration of vehicle or alisertib $(20 \mathrm{mg} / \mathrm{kg}$ twice daily) at 24, 48 and 72 hours post infection if the tumor was not being harvested on that day. Tumors were harvested at 48, 72 and 96 hours post infection and then assayed for virus titer using the standard plaque assay as previous described [55].

\section{Cell infiltrate studies}

When the tumors reached a mean volume $>400$ $\mathrm{mm}^{3}$, the mice were randomized into four study groups: PBS + vehicle, PBS + alisertib, HSV1716 + vehicle and HSV1716 + alisertib. A single dose of PBS or HSV1716 was injected intratumorally on day 0 followed by oral gavage administration of vehicle or alisertib at 24 hours and 48 hours post infection. Tumors and spleens were harvested at 72 hours post infection and then prepared for flow cytometric analysis. Briefly, fresh whole spleens were transferred to $2 \mathrm{~mL}$ PBS and then smashed through a $70 \mu \mathrm{M}$ cell strainer with $5 \mathrm{~mL}$ syringe plunger. Whole tumors were transferred to 6-well dishes containing $2 \mathrm{~mL}$ PBS and then processed as previously described [56]. Briefly, tumors were minced by mechanical chopping and incubated in PBS containing $25 \mu \mathrm{g} / \mathrm{mL}$ liberase blendzyme (Roche Diagnostics., Indianapolis, IN) and $150 \mu \mathrm{g} / \mathrm{mL}$ DNAse I for 1 hour at $37^{\circ} \mathrm{C}$. Tumor samples were then passed through a $70 \mu \mathrm{M}$ cell strainer with the aid of a 5 $\mathrm{mL}$ syringe plunger. After washing splenocytes and tumor cell suspensions once with PBS, the cell suspensions were cleared of red blood cells (RBCs) with ACK lysing buffer (Lonza, Walkersville, MD). The cells were then blocked with 5\% mouse Fc blocking reagent (Miltenyi Biotec Inc., San Diego, CA) in FACS buffer (1\% FBS and 1mM EDTA in PBS) and analyzed by flow cytometry.

\section{Western blot}

S462TY or SK-N-AS cell cultures in 6-well dishes were treated with HSV1716 (MOI =0.5), alisertib (50 $\mathrm{nM}$ ) or both for $24 \mathrm{hr}$. Cells were washed then lysed with Cell Lysis Buffer (Cell Signaling Technology, Danvers, MA) supplemented with Halt Protease Inhibitor Cocktail (Thermo Fisher Scientific, Waltham, MA). Cell lysates were quantified then heat denatured using $10 \mu \mathrm{g}$ protein loaded per lane in a 4-12\% gradient bis-tris gel (Invitrogen, Carlsbad, CA). Protein was transferred to a nitrocellulose membrane and blotted with antibodies against polyADP-ribose polymerase (Roche, Indianapolis, IN), ICP4 (Virusys, Taneytown, MD), and GAPDH (Cell Signaling Technology, Danvers, MA). Densitometry analysis was performed using ImageJ software (NIH, Bethesda, MD). 


\section{Quantitation of apoptotic cells}

Sub-confluent S462TY or SK-N-AS cell cultures in 6-well dishes were treated with HSV1716-GFP (MOI = $0.5)$, alisertib (50 nM) or both for 72 hours. Cells were then harvested, washed twice with cold PBS, stained using the Annexin (APC)/Propidium Iodide (PI) kit according to the manufacturer's instructions (BioLegend, San Diego, $\mathrm{CA}$ ), and analyzed by flow cytometry for the presence of apoptotic cells. Apoptotic cells were gated on annexin positive, HSV1716-GFP-negative cells to include all early and late apoptotic HSV-negative cells.

\section{Statistics}

Plot generation and data analysis was performed using GraphPad Prism Version 6.0 (La Jolla, CA). Statistical significance amongst groups for in vitro assays and cell infiltration studies was determined by two-way analysis of variance (ANOVA). Log-rank test was used to determine significance for survival studies. Significance is reported as $*(P<0.05), * *(P<0.01) . P$ values $<0.05$ were considered significant. In vivo synergy was determined using the Bliss Independence Model [57].

\section{Flow cytometry}

For cellular infiltrate studies, $1 \times 10^{6}$ cells were stained with antibodies against CD49b, F4/80, Ly6G, $\mathrm{CD} 11 \mathrm{~b}$ and $\mathrm{Ly} 6 \mathrm{C}$ according to standard surface staining protocol. Stained cells were then fixed in $0.5 \%$ paraformaldehyde and data acquired on a $\mathrm{BD}$ FASC LSR II (BD, Franklin Lakes, NJ). For apoptotic cell quantitation, cells were processed as described in "quantitation of apoptotic cells" section and then data acquired on a BD FASC LSR II. Further analysis for both studies was carried out using the FlowJo software, version 10.0.3 (Tree Star Inc., Ashland, OR).

\section{Abbreviations}

ANOVA: Analysis of variance; CD11b: cluster of differentiation 11b; CD49b: cluster of differentiation 49b; CR: Complete response; DAB: 3,3'-diaminobenzidine; DMEM: Dulbecco's modified essential media; DNase: deoxyribonuclease; EIF2 $\alpha$ : Eukaryotic translation initiation factor 2, subunit 1 alpha; EDTA: Ethylenediaminetetraacetic acid; FBS: Fetal Bovine Serum; GAPDH: Glyceraldehyde 3-phosphate dehydrogenase; GFP: Green Fluorescent Protein; gMDSCs: Granulocytic myeloid derived suppressor cells; HDAC: Histone deacetylase; HMGB1: High mobility group box 1 protein; HSV1716: Herpes Simplex Virus 1716; ICP4: Infected Cell Protein 4; ICP34.5: Infected
Cell Protein 34.5; Ly6c: lymphocyte antigen 6 complex, locus C1; Ly6g: lymphocyte antigen 6 complex, locus G; MAP kinase: Mitogen-activated protein kinase; mMDSCs: Monocytic myeloid derived suppressor cells; MOI: Multiplicity of Infection; MPNST: Malignant Peripheral Nerve Sheath Tumor; MYCN: v-myc avian myelocytomatosis viral oncogene; NK: Natural Killer; NuMA: nuclear mitotic apparatus protein 1; oHSV: Oncolytic Herpes Simplex Virus; PARP: Poly ADP ribose polymerase; PBS: Phosphate Buffered Saline; PD: Progressive disease; PFU: Plaque forming unit; PKR: Protein kinase R; PR: Partial response; RBC: Red blood cell; SD: Stable disease; UV: Ultraviolet; VITA: virusinduced therapeutic adjuvant.

\section{ACKNOWLEDGMENTS}

We thank Duo Chen for technical assistance. We thank Dr. Winfried Just (Ohio University, Athens, OH) for helpful consultation regarding Bliss Independence Modeling of in vivo synergy.

\section{CONFLICTS OF INTEREST}

JC is an employee of Virttu Biologics Ltd. JE is an employee of Takeda Pharmaeuticals. The rest of the authors declare no conflict of interest.

\section{FUNDING}

This work was supported by Nationwide Children's Hospital, the National Institutes of Health (1R21NS084885-02), the Ohio State University's College of Medicine NIH training grant (4T32GM068412-09), and by CancerFree Kids, Pediatric Cancer Research Alliance (Cincinnati, Ohio). Alisertib used in this study was provided by ${ }^{4}$ Takeda Pharmaceuticals International Co, (Boston, MA). Oncolytic HSV HSV1716 used in the study was provided by Virttu Biologics, Ltd (Glasgow, UK).

\section{Author contributions}

Data collection, writing, editing: MAC, LS, TAR, BN, CYC, PYW, BJH, KAS, NR, TPC.

Data collection, editing: AVP.

Writing, editing: JAE, JC.

Data collection: MRF, KEC.

\section{REFERENCES}

1. Nikonova AS, Astsaturov I, Serebriiskii IG, Dunbrack RL, Jr. and Golemis EA. Aurora A kinase (AURKA) in normal 
and pathological cell division. Cell Mol Life Sci. 2013; 70:661-687.

2. Kettenbach AN, Schweppe DK, Faherty BK, Pechenick D, Pletnev AA and Gerber SA. Quantitative phosphoproteomics identifies substrates and functional modules of Aurora and Polo-like kinase activities in mitotic cells. Sci Signal. 2011; 4:rs5.

3. Maris JM, Morton CL, Gorlick R, Kolb EA, Lock R, Carol H, Keir ST, Reynolds CP, Kang MH, Wu J, Smith MA and Houghton PJ. Initial testing of the aurora kinase A inhibitor MLN8237 by the Pediatric Preclinical Testing Program (PPTP). Pediatr Blood Cancer. 2010; 55:26-34.

4. Patel AV, Eaves D, Jessen WJ, Rizvi TA, Ecsedy JA, Qian MG, Aronow BJ, Perentesis JP, Serra E, Cripe TP, Miller SJ and Ratner N. Ras-driven transcriptome analysis identifies aurora kinase $\mathrm{A}$ as a potential malignant peripheral nerve sheath tumor therapeutic target. Clin Cancer Res. 2012; 18:5020-5030.

5. Meraldi P, Honda R and Nigg EA. Aurora-A overexpression reveals tetraploidization as a major route to centrosome amplification in p53-/- cells. EMBO J. 2002; 21:483-492.

6. Katayama H, Wang J, Treekitkarnmongkol W, Kawai H, Sasai K, Zhang H, Wang H, Adams HP, Jiang S, Chakraborty SN, Suzuki F, Arlinghaus RB, Liu J, et al. Aurora kinase-A inactivates DNA damage induced apoptosis and spindle assembly checkpoint response functions of p73. Can Cell. 2012; 21:196-211.

7. Brown SM, MacLean AR, Aitken JD and Harland J. ICP34.5 influences herpes simplex virus type 1 maturation and egress from infected cells in vitro. J Gen Virol. 1994; 75:3679-3686.

8. Jing X, Cerveny M, Yang K and He B. Replication of herpes simplex virus 1 depends on the gamma 134.5 functions that facilitate virus response to interferon and egress in the different stages of productive infection. J Virol. 2004; 78:7653-7666.

9. Liu B, Robinson M, Han Z-Q, Branston R, English C, Reay P, McGrath Y, Thomas S, Thornton M, Bullock P, Love C and Coffin R. ICP34.5 deleted herpes simplex virus with enhanced oncolytic, immune stimulating, and anti-tumour proporties. Gene Ther. 2003; 10:292-303.

10. Smith KD, Mezhir JJ, Bickenbach K, Veerapong J, Charron J, Posner MC, Roizman B and Weichselbaum RR. Activated MEK suppresses activation of PKR and enables efficient replication and in vivo oncolysis by Deltagamma(1)34.5 mutants of herpes simplex virus 1. J Virol. 2006; 80:11101120.

11. Antoszczyk S, Spyra M, Mautner VF, Kurtz A, StemmerRachamimov AO, Martuza RL, Rabkin SD. Treatment of orthotopic malignant peripheral nerve sheath tumors with oncolytic herpes simplex virus. Neuro Oncol. 2014; 16:1057-66.

12. Wang PY, Swain HM, Kunkler AL, Chen CY, Hutzen BJ, Arnold MA, Streby KA, Collins MH, Dipasquale B,
Stanek JR, Conner J, van Kuppevelt TH, Glorioso JC, et al. Neuroblastomas vary widely in their sensitivities to herpes simplex virotherapy unrelated to virus receptors and susceptibility. Gene Ther. 2016; 23:135-43.

13. Mohan $\mathrm{P}$, Castellsague J, Jiang J, Allen K, Chen H, Nemirovsky O, Spyra M, Hu K, Kluwe L, Pujana MA, Villanueva A, Mautner VF, Keats JJ, et al. Genomic imbalance of HMMR/RHAMM regulates the sensitivity and response of malignant peripheral nerve sheath tumour cells to aurora kinase inhibition. Oncotarget. 2013; 4:80-93. doi: 10.18632/oncotarget.793.

14. Mahller YY, Rangwala F, Ratner $\mathrm{N}$ and Cripe TP. Malignant peripheral nerve sheath tumors with high and low Ras-GTP are permissive for oncolytic herpes simplex virus mutants. Pediatr Blood Cancer. 2006; 46:745-754.

15. Cassady KA, Haworth KB, Jackson J, Markert JM and Cripe TP. To Infection and Beyond: The Multi-Pronged Anti-Cancer Mechanisms of Oncolytic Viruses. Viruses. 2016; 8: pii: E43.

16. MacKie RM, Stewart B and Brown SM. Intralesional injection of herpes simplex virus 1716 in metastatic melanoma. Lancet. 2001; 357:525-526.

17. Papanastassiou V, Rampling R, Fraser M, Petty R, Hadley D, Nicoll J, Harland J, Mabbs R and Brown M. The potential for efficacy of the modified (ICP 34.5(-)) herpes simplex virus HSV1716 following intratumoural injection into human malignant glioma: a proof of principle study. Gene Ther. 2002; 9:398-406.

18. Harrow S, Papanastassiou V, Harland J, Mabbs R, Petty R, Fraser M, Hadley D, Patterson J, Brown SM and Rampling R. HSV1716 injection into the brain adjacent to tumour following surgical resection of high-grade glioma: safety data and long-term survival. Gene Ther. 2004; 11:16481658.

19. Mace AT, Ganly I, Soutar DS and Brown SM. Potential for efficacy of the oncolytic Herpes simplex virus 1716 in patients with oral squamous cell carcinoma. Head Neck. 2008; 30:1045-1051.

20. Mahller YY, Williams JP, Baird WH, Mitton B, Grossheim J, Saeki Y, Cancelas JA, Ratner N and Cripe TP. Neuroblastoma cell lines contain pluripotent tumor initiating cells that are susceptible to a targeted oncolytic virus. PLoS One. 2009; 4:e4235.

21. Carol H, Boehm I, Reynolds CP, Kang MH, Maris JM, Morton CL, Gorlick R, Kolb EA, Keir ST, Wu J, Wozniak AE, Yang Y, Manfredi M, et al. Efficacy and pharmacokinetic/pharmacodynamic evaluation of the Aurora kinase A inhibitor MLN8237 against preclinical models of pediatric cancer. Cancer Chemother Pharmacol. 2011; 68:1291-1304.

22. Stanziale SF, Petrowsky H, Adusumilli PS, Ben-Porat L, Gonen $\mathrm{M}$ and Fong Y. Infection with oncolytic herpes simplex virus-1 induces apoptosis in neighboring human cancer cells: a potential target to increase anticancer activity. Clin Cancer Res. 2004; 10:3225-3232. 
23. Takasu A, Masui A, Hamada M, Imai T, Iwai S and Yura Y. Immunogenic cell death by oncolytic herpes simplex virus type 1 in squamous cell carcinoma cells. Cancer Gene Ther. 2016; 23:107-113.

24. Niu H, Manfredi M and Ecsedy JA. Scientific Rationale Supporting the Clinical Development Strategy for the Investigational Aurora A Kinase Inhibitor Alisertib in Cancer. Front Oncol. 2015; 5:189.

25. Durlacher CT, Li ZL, Chen XW, He ZX and Zhou SF. An update on the pharmacokinetics and pharmacodynamics of alisertib, a selective Aurora kinase A inhibitor. Clinical and experimental pharmacology \& physiology. 2016; 43:585601.

26. Faisal A, Vaughan L, Bavetsias V, Sun C, Atrash B, Avery S, Jamin Y, Robinson SP, Workman P, Blagg J, Raynaud FI, Eccles SA, Chesler L, et al. The aurora kinase inhibitor CCT137690 downregulates MYCN and sensitizes MYCNamplified neuroblastoma in vivo. Mol Cancer Ther. 2011; 10:2115-2123.

27. Gustafson WC, Meyerowitz JG, Nekritz EA, Chen J, Benes C, Charron E, Simonds EF, Seeger R, Matthay KK, Hertz NT, Eilers M, Shokat KM and Weiss WA. Drugging MYCN through an allosteric transition in Aurora kinase A. Cancer Cell. 2014; 26:414-427.

28. DuBois SG, Marachelian A, Fox E, Kudgus RA, Reid JM, Groshen S, Malvar J, Bagatell R, Wagner L, Maris JM, Hawkins R, Courtier J, Lai H, et al. Phase I Study of the Aurora A Kinase Inhibitor Alisertib in Combination With Irinotecan and Temozolomide for Patients With Relapsed or Refractory Neuroblastoma: A NANT (New Approaches to Neuroblastoma Therapy) Trial. J Clin Oncol. 2016; 34:1368-1375.

29. Mosse YP, Lipsitz E, Fox E, Teachey DT, Maris JM, Weigel B, Adamson PC, Ingle MA, Ahern CH and Blaney SM. Pediatric phase I trial and pharmacokinetic study of MLN8237, an investigational oral selective small-molecule inhibitor of Aurora kinase A: a Children's Oncology Group Phase I Consortium study. Clin Cancer Res. 2012; 18:60586064.

30. Dickson MA, Mahoney MR, Tap WD, D’Angelo SP, Keohan ML, Van Tine BA, Agulnik M, Horvath LE, Nair JS and Schwartz GK. Phase II study of MLN8237 (Alisertib) in advanced/metastatic sarcoma. Ann Oncol. 2016; 27:1855-60.

31. Andtbacka RH, Kaufman HL, Collichio F, Amatruda T, Senzer N, Chesney J, Delman KA, Spitler LE, Puzanov I, Agarwala SS, Milhem M, Cranmer L, Curti B, et al. Talimogene Laherparepvec Improves Durable Response Rate in Patients With Advanced Melanoma. J Clin Oncol. 2015; 33:2780-8.

32. Braekeveldt N, Wigerup C, Gisselsson D, Mohlin S, Merselius $\mathrm{M}$, Beckman $\mathrm{S}$, Jonson $\mathrm{T}$, Borjesson A, Backman T, Tadeo I, Berbegall AP, Ora I, Navarro S, et al. Neuroblastoma patient-derived orthotopic xenografts retain metastatic patterns and geno- and phenotypes of patient tumours. Int J Cancer. 2015; 136:E252-261.

33. Mahller YY, Vaikunth SS, Ripberger MC, Baird WH, Saeki Y, Cancelas JA, Crombleholme TM and Cripe TP. Tissue inhibitor of metalloproteinase-3 via oncolytic herpesvirus inhibits tumor growth and vascular progenitors. Cancer Res. 2008; 68:1170-1179.

34. Jackson JD, McMorris AM, Roth JC, Coleman JM, Whitley RJ, Gillespie GY, Carroll SL, Markert JM and Cassady KA. Assessment of oncolytic HSV efficacy following increased entry-receptor expression in malignant peripheral nerve sheath tumor cell lines. Gene Ther. 2014; 21:984-990.

35. Jackson JD, Markert JM, Li L, Carroll SL and Cassady KA. STAT1 and NF-kappaB Inhibitors Diminish Basal Interferon-Stimulated Gene Expression and Improve the Productive Infection of Oncolytic HSV in MPNST Cells. Mol Cancer Res. 2016; 14:482-492.

36. Alvarez-Breckenridge CA, Yu J, Price R, Wojton J, Pradarelli J, Mao H, Wei M, Wang Y, He S, Hardcastle J, Fernandez SA, Kaur B, Lawler SE, et al. NK cells impede glioblastoma virotherapy through NKp30 and NKp46 natural cytotoxicity receptors. Nat Med. 2012; 18:18271834.

37. Kumar V, Patel S, Tcyganov E and Gabrilovich DI. The Nature of Myeloid-Derived Suppressor Cells in the Tumor Microenvironment. Trends Immunol. 2016; 37:208-220.

38. Woller N, Gurlevik E, Fleischmann-Mundt B, Schumacher A, Knocke S, Kloos AM, Saborowski M, Geffers R, Manns MP, Wirth TC, Kubicka S and Kuhnel F. Viral Infection of Tumors Overcomes Resistance to PD-1-immunotherapy by Broadening Neoantigenome-directed T-cell Responses. Mol Ther. 2015; 23:1630-1640.

39. Rajani KR and Vile RG. Harnessing the Power of OncoImmunotherapy with Checkpoint Inhibitors. Viruses. 2015; 7:5889-5901.

40. Blas-Rus N, Bustos-Moran E, Perez de Castro I, de Carcer G, Borroto A, Camafeita E, Jorge I, Vazquez J, Alarcon B, Malumbres M, Martin-Cofreces NB and Sanchez-Madrid F. Aurora A drives early signalling and vesicle dynamics during T-cell activation. Nature communications. 2016; 7:11389.

41. Vilgelm AE, Johnson CA, Prasad N, Yang J, Chen SC, Ayers GD, Pawlikowski JS, Raman D, Sosman JA, Kelley M, Ecsedy JA, Shyr Y, Levy SE, et al. Connecting the Dots: Therapy-Induced Senescence and a Tumor-Suppressive Immune Microenvironment. J Natl Cancer Inst. 2016; 108:djv406.

42. Iankov ID, Kurokawa $\mathrm{CB}$, D'Assoro $\mathrm{AB}$, Ingle JN, Domingo-Musibay E, Allen C, Crosby CM, Nair AA, Liu MC, Aderca I, Federspiel MJ and Galanis E. Inhibition of the Aurora A kinase augments the anti-tumor efficacy of oncolytic measles virotherapy. Cancer Gene Ther. 2015; 22:438-444. 
43. Igase M, Hwang CC, Kambayashi S, Kubo M, Coffey M, Miyama TS, Baba K, Okuda M, Noguchi S and Mizuno T. Oncolytic reovirus synergizes with chemotherapeutic agents to promote cell death in canine mammary gland tumor. Can J Vet Res. 2016; 80:21-31.

44. McKenzie BA, Zemp FJ, Pisklakova A, Narendran A, McFadden G, Lun X, Kenchappa RS, Kurz EU and Forsyth PA. In vitro screen of a small molecule inhibitor drug library identifies multiple compounds that synergize with oncolytic myxoma virus against human brain tumorinitiating cells. Neuro Oncol. 2015; 17:1086-1094.

45. Toughiri R, Li X, Du Q and Bieberich CJ. Phosphorylation of NuMA by Aurora-A kinase in PC-3 prostate cancer cells affects proliferation, survival, and interphase NuMA localization. J Cell Biochem. 2013; 114:823-830.

46. Yamauchi Y, Kiriyama K, Kimura H and Nishiyama Y. Herpes simplex virus induces extensive modification and dynamic relocalisation of the nuclear mitotic apparatus (NuMA) protein in interphase cells. Journal of cell science. 2008; 121:2087-2096.

47. Yoo JY, Hurwitz BS, Bolyard C, Yu JG, Zhang J, Selvendiran K, Rath KS, He S, Bailey Z, Eaves D, Cripe TP, Parris DS, Caligiuri MA, et al. Bortezomib-induced unfolded protein response increases oncolytic HSV-1 replication resulting in synergistic antitumor effects. Clin Cancer Res. 2014; 20:3787-3798.

48. Bolyard C, Yoo JY, Wang PY, Saini U, Rath KS, Cripe TP, Zhang J, Selvendiran K and Kaur B. Doxorubicin synergizes with 34.5ENVE to enhance antitumor efficacy against metastatic ovarian cancer. Clin Cancer Res. 2014; 20:6479-6494.

49. Yoo JY, Pradarelli J, Haseley A, Wojton J, Kaka A, Bratasz A, Alvarez-Breckenridge CA, Yu JG, Powell K, Mazar AP, Teknos TN, Chiocca EA, Glorioso JC, et al. Copper chelation enhances antitumor efficacy and systemic delivery of oncolytic HSV. Clin Cancer Res. 2012; 18:4931-4941.

50. Katsura T, Iwai S, Ota Y, Shimizu H, Ikuta K and Yura Y. The effects of trichostatin A on the oncolytic ability of herpes simplex virus for oral squamous cell carcinoma cells. Cancer Gene Ther. 2009; 16:237-245.

51. Otsuki A, Patel A, Kasai K, Suzuki M, Kurozumi K,
Chiocca EA and Saeki Y. Histone deacetylase inhibitors augment antitumor efficacy of herpes-based oncolytic viruses. Mol Ther. 2008; 16:1546-1555.

52. McKie EA, MacLean AR, Lewis AD, Cruickshank G, Rampling R, Barnett SC, Kennedy PG and Brown SM. Selective in vitro replication of herpes simplex virus type 1 (HSV-1) ICP34.5 null mutants in primary human CNS tumours--evaluation of a potentially effective clinical therapy. Br J Cancer. 1996; 74:745-752.

53. Randazzo BP, Kucharczuk JC, Litzky LA, Kaiser LR, Brown SM, MacLean A, Albelda SM and Fraser NW. Herpes simplex 1716--an ICP 34.5 mutant--is severely replication restricted in human skin xenografts in vivo. Virology. 1996; 223:392-395.

54. Chou TC and Talalay P. Generalized equations for the analysis of inhibitions of Michaelis-Menten and higherorder kinetic systems with two or more mutually exclusive and nonexclusive inhibitors. Eur J Biochem. 1981; 115:207216.

55. Eshun FK, Currier MA, Gillespie RA, Fitzpatrick JL, Baird WH and Cripe TP. VEGF blockade decreases the tumor uptake of systemic oncolytic herpes virus but enhances therapeutic efficacy when given after virotherapy. Gene Ther. 2010; 17:922-929.

56. Currier MA, Eshun FK, Sholl A, Chernoguz A, Crawford K, Divanovic S, Boon L, Goins WF, Frischer JS, Collins MH, Leddon JL, Baird WH, Haseley A, et al. VEGF blockade enables oncolytic cancer virotherapy in part by modulating intratumoral myeloid cells. Mol Ther. 2013; 21:1014-1023.

57. Zhao W, Sachsenmeier K, Zhang L, Sult E, Hollingsworth RE and Yang H. A New Bliss Independence Model to Analyze Drug Combination Data. J Biomol Screen. 2014; 19:817-821. 